\title{
Regular spanning subgraphs of bipartite graphs of high minimum degree
}

\author{
Béla Csaba* ${ }^{* \dagger}$ \\ Submitted: Aug 1, 2007; Accepted: Oct 5, 2007; Published: Oct 16, 2007 \\ Mathematics Subject Classification: 05C70
}

\begin{abstract}
Let $G$ be a simple balanced bipartite graph on $2 n$ vertices, $\delta=\delta(G) / n$, and $\rho_{0}=\frac{\delta+\sqrt{2 \delta-1}}{2}$. If $\delta \geq 1 / 2$ then $G$ has a $\left\lfloor\rho_{0} n\right\rfloor$-regular spanning subgraph. The statement is nearly tight.
\end{abstract}

\section{Introduction}

In this paper we will consider regular spanning subgraphs of simple graphs. We mostly use standard graph theory notation: $V(G)$ and $E(G)$ will denote the vertex and the edge set of a graph $G$, respectively. The degree of $x \in V(G)$ is denoted by $\operatorname{deg}_{G}(x)$ (we may omit the subscript), $\delta(G)$ is the minimum degree of $G$. We call a bipartite graph $G(A, B)$ with color classes $A$ and $B$ balanced if $|A|=|B|$. For $X, Y \subset V(G)$ we denote the number of edges of $G$ having one endpoint in $X$ and the other endpoint in $Y$ by $e(X, Y)$. If $T \subset V(G)$ then $\left.G\right|_{T}$ denotes the subgraph we get after deleting every vertex of $V-T$ and the edges incident to them. Finally, $K_{r, s}$ is the complete bipartite graph on color classes of size $r$ and $s$ for two positive integers $r$ and $s$.

If $f: V(H) \rightarrow \mathrm{Z}^{+}$is a function, then an $f$-factor is a subgraph $H^{\prime}$ of the graph $H$ such that $\operatorname{deg}_{H^{\prime}}(x)=f(x)$ for every $x \in V(H)$. Notice, that when $f \equiv r$ for some $r \in \mathrm{Z}^{+}$, then $H^{\prime}$ is an $r$-regular subgraph of $H$.

There are several results concerning $f$-factors of graphs. Perhaps the most notable among them is the theorem of Tutte [7]. Finding $f$-factors is in general not an easy task even for the case $f$ is a constant and the graph is regular (see eg., [1]). In this paper we look for $f$-factors in (not necessarily regular) bipartite graphs with large minimum degree, for $f \equiv r$.

\footnotetext{
*Analysis and Stochastics Research Group of the Hungarian Academy of Sciences, University of Szeged, Hungary and Department of Mathematics, Western Kentucky University, Bowling Green, KY, USA

${ }^{\dagger}$ Partially supported by OTKA T049398 and by Hungarian State Eötvös Fellowship.
} 
Theorem 1 Let $G(A, B)$ be a balanced bipartite graph on $2 n$ vertices, and assume that $\delta=\delta(G) / n \geq 1 / 2$. Set $\rho_{0}=\frac{\delta+\sqrt{2 \delta-1}}{2}$. Then

(I) $G$ has a $\left.\rho_{0} n\right\rfloor$-regular spanning subgraph;

(II) moreover, for every $\delta>1 / 2$ if $n$ is sufficiently large and $\delta n$ is an integer then there exists a balanced bipartite graph $G_{\delta}$ having minimum degree $\delta$ such that it does not admit a spanning regular subgraph of degree larger than $\left\lceil\rho_{0} n\right\rceil$.

The above theorem plays a crucial role in the proof of some results in extremal graph theory $([2,3])$.

\section{The main tool}

Let $F$ be a bipartite graph with color classes $A$ and $B$. By the well-known König-Hall theorem there is a perfect matching in $F$ if and only if $|N(S)| \geq|S|$ for every $S \subset A$. We are going to need a far reaching generalization of this result, due to Gale and Ryser $[6,4]$ (one can find the proof in [5] as well). It gives a necessary and sufficient condition for the existence of an $f$-factor in a bipartite graph:

Proposition 2 Let $F$ be a bipartite graph with bipartition $\{A, B\}$, and $f(x) \geq 0$ an integer valued function on $A \cup B$. $F$ has an $f$-factor if and only if

$$
\text { (i) } \sum_{x \in A} f(x)=\sum_{y \in B} f(y)
$$

and

$$
\text { (ii) } \sum_{x \in X} f(x) \leq e(X, Y)+\sum_{y \in B-Y} f(y)
$$

for all $X \subset A$ and $Y \subset B$.

\section{Proof of Theorem 1}

We will show the two parts of the theorem in separate subsections.

\subsection{Proof of part $I$}

Observe, that since we are looking for a spanning regular subgraph, the $f$ function of Proposition 2 will be identically $\rho$ for some constant $\rho$. We start with some notation: for $X \subset A$ let $\xi=|X| / n$, and for $Y \subset B$ let $\sigma=|Y| / n$. We will normalize $e(X, Y)$ : $\eta(X, Y)=e(X, Y) / n^{2}$. Let

$$
\eta_{m}(\xi, \sigma)=\min \{\eta(X, Y): X \subset A, Y \subset B,|X| / n=\xi,|Y| / n=\sigma\}
$$


Since $f$ is identically $\rho n$, condition $(i)$ of Proposition 2 is satisfied. Moreover, if $\rho n$ is an integer and

$$
\rho(\xi+\sigma-1) \leq \eta_{m}(\xi, \sigma)
$$

for some $\rho$ and for every $0 \leq \xi, \sigma \leq 1$, then (ii) is satisfied, hence, $G$ has a $\rho n$-regular spanning subgraph. In the rest of this section we will show that the above inequality is valid for $\rho=\left\lfloor\rho_{0} n\right\rfloor / n$.

Clearly, $e(X, Y) \geq|X|(\delta n-|B-Y|)$ and $e(X, Y) \geq|Y|(\delta n-|A-X|)$ for arbitrary sets $X \subset A$ and $Y \subset B$. Hence, we have that $\eta_{m}(\xi, \sigma) \geq \max (\xi(\delta+\sigma-1), \sigma(\delta+\xi-1))$. (In fact we always have that $\eta_{m} \geq 0$, since it is the edge density between the two color classes in $G$.)

First consider the case $\xi=\sigma$. We are looking for a $\rho$ for which $\rho(2 \xi-1) \leq \xi(\delta+\xi-1)$. In another form, we need that

$$
p_{\rho}(\xi)=\xi^{2}+(\delta-2 \rho-1) \xi+\rho \geq 0 .
$$

The discriminant of the above polynomial is the polynomial $d \operatorname{cr}(\rho)=4 \rho^{2}-4 \delta \rho+\delta^{2}-$ $2 \delta+1$. Clearly, if $d c r(\rho) \leq 0$ for some $\rho$, then $p_{\rho}(\xi) \geq 0$.

One can directly find the roots of $d \operatorname{cr}(\rho): \frac{\delta \pm \sqrt{2 \delta-1}}{2}$. At this point we have to be careful, since the degrees in a graph are non-negative integers, so $\rho n$ has to be a natural number. We will show that $d c r(\rho) \leq 0$ for $\rho=\lfloor(\delta+\sqrt{2 \delta-1}) n / 2\rfloor / n$.

Clearly, $d \operatorname{cr}(x) \leq 0$ in $I=[(\delta-\sqrt{2 \delta-1}) / 2,(\delta+\sqrt{2 \delta-1}) / 2]$, the length of this interval is $\sqrt{2 \delta-1}$. Divide the $[0,1]$ interval into $n$ disjoint subintervals each of length $1 / n$, denote the set of the endpoints of these subintervals by $S$. Observe that is $I \cap S \geq 1$, then we can pick the largest point of this intersection, this is $\rho \in I$, and we are done with proving that $p_{\rho}(\xi) \geq 0$.

We will investigate two cases: first, if $\delta>1 / 2$, and second, if $\delta=1 / 2$.

First case: $\delta>1 / 2$. We know that $\delta n$ is an integer, it is larger than $n / 2$, hence, $\delta n \geq \frac{n+1}{2}$. If the length of $I$ is at least $1 / n$, it will intersect with $S$. Assuming that $\sqrt{2 \delta-1}<\frac{1}{n}$ we would get $\delta n<\frac{n^{2}+1}{2 n}$, but the latter expression is less than $\frac{n+1}{2}$. Hence, in this case $|I \cap S| \geq 1$.

Let $g(\xi, \sigma)=\sigma(\delta+\xi-1)-\rho(\xi+\sigma-1)$. We will show, that $g(\xi, \sigma) \geq 0$ for $0 \leq \sigma \leq \xi \leq 1$, in the lower right triangle $T$ of the unit square. This will prove that $(i i)$ of Proposition 2 is satisfied. Notice, that $g$ is bounded in the triangle above, $-2 \leq g(\xi, \sigma) \leq \eta_{m}(\xi, \sigma)-$ $\rho(\xi+\sigma-1)$, and continously differentiable.

Let us check the sign of $g$ on the border of the triangle. Since $\rho=\lfloor(\delta+\sqrt{2 \delta-1}) n / 2\rfloor / n$, we have that $g(\xi, \xi) \geq 0 . g(\xi, 0)=-\rho(\xi-1) \geq 0$, and $g(1, \sigma)=\sigma(\delta-\rho) \geq 0$, because $\delta \geq(\delta+\sqrt{2 \delta-1}) / 2$. Let us check the partial derivatives of $g$ :

$$
\frac{\partial g}{\partial \xi}=\sigma-\rho
$$

and

$$
\frac{\partial g}{\partial \sigma}=\delta+\xi-1-\rho
$$


Assuming that $g$ achieves its minimum inside the triangle at the point $\left(\xi^{\prime}, \sigma^{\prime}\right)$ the partial derivatives of $g$ have to diminish at $\left(\xi^{\prime}, \sigma^{\prime}\right)$. It would then follow that $\sigma^{\prime}=\rho$ and $\xi^{\prime}=$ $1+\rho-\delta$, therefore, $g\left(\xi^{\prime}, \sigma^{\prime}\right)=\rho^{2}-\rho(2 \rho-\delta)=\delta \rho-\rho^{2}$. Hence $g$ is non-negative in $T$. The same reasoning works for the triangle $0 \leq \xi \leq \sigma \leq 1$, this follows easily by symmetry. With this we finished the proof for the case $\delta>1 / 2$.

Second case: $\delta=1 / 2$. If $\delta n$ is even ( $n$ is divisible by 4 ), we are done, since in this case $I$ contains the point $\delta / 2=\lfloor n / 4\rfloor / n$, and $\delta n / 2$ is an integer. Therefore we have that $p_{1 / 4}(\xi) \geq 0$, and as above, one can check that $g$ is non-negative in every point of $T$.

There is only one case left: if $\delta n$ is odd, that is, $n$ is of the form $4 k+2$ for some natural number $k$. In this case we want to prove, that that the spanning subgraph is $\frac{k}{4 k+2}$-regular.

First observe, that for our purposes it is sufficient if $g(\xi, \sigma) \geq 0$ in a discrete point set: in the points $(\xi, \sigma)$ belonging to $(S \times S) \cap T$, since $|X|$ and $|Y|$ are natural numbers. Set $\rho=\frac{k}{4 k+2}$ and analyze the polynomial $p_{\rho}(\xi)$. It is an easy exercise to check that it has two distinct roots: $1 / 2=\frac{2 k+1}{4 k+2}$ and $1 / 2-1 / n=\frac{2 k}{4 k+2}$. Hence, $p_{\rho}(\xi) \geq 0$ for $\xi \notin(1 / 2-1 / n, 1 / 2)$.

We will cut out a small open triangle $T_{s}$ from $T$. $T_{s}$ has vertices $(1 / 2,1 / 2),(1 / 2,1 / 2-$ $1 / n)$ and $(1 / 2-1 / n, 1 / 2-1 / n)$. Clearly, $T-T_{s}$ is closed and $T_{s} \cap(S \times S)=\emptyset$.

Recall, that $g(\xi, \sigma)=\sigma(\delta+\xi-1)-\rho(\xi+\sigma-1)$ for $0 \leq \sigma \leq \xi \leq 1$. We will check the sign of $g$ on the border of $T-T_{s}$. There are two line segments for which we cannot apply our earlier results concerning $g$. The first is

$$
L_{1}=\left\{(\xi, \sigma): \frac{n-2}{2 n} \leq \xi \leq \frac{1}{2}, \sigma=\frac{n-2}{2 n}\right\}
$$

the second is

$$
L_{2}=\left\{(\xi, \sigma): \xi=\frac{1}{2}, \frac{n-2}{2 n} \leq \sigma \leq \frac{1}{2}\right\}
$$

On $L_{1}$ we get that

$$
g\left(\xi, \frac{n-2}{2 n}\right)=\frac{n-2}{2 n}\left(\xi-\frac{1}{2}\right)-\frac{k}{4 k+2}\left(\xi-\frac{n-2}{2 n}\right)=\frac{k}{n} \xi-\frac{k}{2 n}+\frac{k}{n^{2}} .
$$

It is easy to see that the above expression is non-negative for every $(n-2) /(2 n) \leq \xi \leq 1 / 2$.

For $L_{2}$ we have

$$
g\left(\frac{1}{2}, \sigma\right)=\sigma\left(\frac{1}{2}+\frac{1}{2}-1\right)-\frac{k}{4 k+2}\left(\frac{1}{2}+\sigma-1\right)=\frac{k}{4 k+2}\left(\frac{1}{2}-\sigma\right) \geq 0
$$

for $(n-2) /(2 n) \leq \sigma \leq 1 / 2$.

In order to finish proving that $g$ is non-negative in every point of $(S \times S) \cap\left(T-T_{s}\right)$ it is sufficient to show that the minimum of $g$ inside $T-T_{s}$ is at least as large as the minimum of $g$ on the border of $T-T_{s}$. This can be shown along the same lines as previously. By symmetry we will get that condition $(i i)$ of Proposition 2 is satisfied in every point of $S \times S$. 


\subsection{Proof of part $I I$}

For proving part $I I$ of the theorem we want to construct a class of balanced bipartite graphs the elements of which cannot have a large regular spanning subgraph. We will achieve this goal in two steps. First, we will consider a simple linear function, which, as we will see later, is closely related to our task. In the second step we will construct those bipartite graph which satisfy part $I I$ of Theorem 1 .

Set $\gamma^{\prime}=\frac{1-\sqrt{2 \delta-1}}{2}$ and let $0<p<1$. Consider the following equation:

$$
(1-p)\left(1-\gamma^{\prime}\right)=\gamma^{\prime}(1-p)+\delta-\gamma^{\prime}
$$

It is easy to see that $p^{\prime}=\frac{\delta+\gamma^{\prime}-1}{2 \gamma^{\prime}-1}$ is its solution. We have that

$$
\left(1-p^{\prime}\right)\left(1-\gamma^{\prime}\right)=\left(1-\frac{\delta+\gamma^{\prime}-1}{2 \gamma^{\prime}-1}\right)\left(1-\gamma^{\prime}\right)=\frac{\gamma^{\prime}-\delta}{2 \gamma^{\prime}-1}\left(1-\gamma^{\prime}\right)
$$

Substituting $\gamma^{\prime}=\frac{1-\sqrt{2 \delta-1}}{2}$ we get

$$
\begin{gathered}
\frac{\delta-\frac{1-\sqrt{2 \delta-1}}{2}}{\sqrt{2 \delta-1}}\left(1-\frac{1-\sqrt{2 \delta-1}}{2}\right)=\frac{2 \delta-1+\sqrt{2 \delta-1}}{\sqrt{2 \delta-1}} \frac{1+\sqrt{2 \delta-1}}{2}= \\
\frac{1+\sqrt{2 \delta-1} \frac{1+\sqrt{2 \delta-1}}{2}=\frac{\delta+\sqrt{2 \delta-1}}{2} .}{2}
\end{gathered}
$$

We promised to define a class of bipartite graphs for $\delta>1 / 2$ which exist for every sufficiently large value of $n$ if $\delta n$ is a natural number, such that these graphs do not admit spanning regular graphs with large degree.

For that let $\gamma=\left\lceil\gamma^{\prime} n\right\rceil / n$. Then $\gamma n$ is an integer, and $\gamma^{\prime} \leq \gamma \leq \gamma^{\prime}+1 / n$. Let $G=(A, B, E)$ be a balanced bipartite graph on $2 n$ vertices. $A$ is divided into two disjoint subsets, $A_{l}$ and $A_{e}$, we also divide $B$ into $B_{l}$ and $B_{e}$. We will have that $\left|A_{l}\right|=\left|B_{l}\right|=\gamma n$ and $\left|A_{e}\right|=\left|B_{e}\right|=(1-\gamma) n$. There are no edges in between the vertices of $A_{l}$ and $B_{l}$. The subgraphs $\left.G\right|_{A_{l} \cup B_{e}}$ and $\left.G\right|_{B_{l} \cup A_{e}}$ are isomorphic to $K_{\gamma n,(1-\gamma) n}$, therefore, every vertex in $A_{l} \cup B_{l}$ has degree $(1-\gamma) n$. We require that every vertex in $A_{e} \cup B_{e}$ has degree $\delta n$, hence, $\left.G\right|_{A_{e} \cup B_{e}}$ will be a $(\delta-\gamma) n$-regular graph. Observe, that $\gamma<\delta<1-\gamma$, thus, $\delta(G)=\delta n$.

Let us consider a simple method for edge removal from $G$ : given $0<p<1$ discard $p(1-\gamma) n$ incident edges for every vertex in $A_{l} \cup B_{l}$, and no edge from $\left.G\right|_{A_{e} \cup B_{e}}$. Of course, we need that $p(1-\gamma) n$ is an integer.

Then a vertex in $A_{l} \cup B_{l}$ will have degree $(1-p)(1-\gamma) n$, and the average degree of the vertices of $A_{e} \cup B_{e}$ will be $\gamma(1-p) n+(\delta-\gamma) n$. Choose $\widetilde{p}$ to be the solution of the following equation:

$$
(1-p)(1-\gamma) n=\gamma(1-p) n+(\delta-\gamma) n
$$

Notice, that the only difference between (1) and (2) is that we subsituted $\gamma^{\prime}$ by $\gamma$. One can see that if $p<\tilde{p}$ then there is a vertex $x \in A_{e} \cup B_{e}$ such that every vertex of $A_{l} \cup B_{l}$ 
will have degree larger than $\operatorname{deg}(x)$. That is, for finding a regular subgraph more edges have to be discarded among those which are incident to the vertices of $A_{l} \cup B_{l}$.

The solution of (2) is $\widetilde{p}=\frac{\delta+\gamma-1}{2 \gamma-1}$ (here $\widetilde{p}(1-\gamma) n$ is not necessarily an integer). Computing the derivative shows that $\gamma \geq \gamma^{\prime}$ implies $p^{\prime} \geq \tilde{p}$. Let us show that $p^{\prime}-\tilde{p}$ is small:

$$
\begin{gathered}
p^{\prime}-\widetilde{p}=\frac{\delta+\gamma^{\prime}-1}{2 \gamma^{\prime}-1}-\frac{\delta+\gamma-1}{2 \gamma-1}= \\
\frac{\left(\delta+\gamma^{\prime}-1\right)(2 \gamma-1)-(\delta+\gamma-1)\left(2 \gamma^{\prime}-1\right)}{(2 \gamma-1)\left(2 \gamma^{\prime}-1\right)}=\frac{2 \gamma \delta-2 \gamma^{\prime} \delta+\gamma^{\prime}-\gamma}{(2 \gamma-1)\left(2 \gamma^{\prime}-1\right)} .
\end{gathered}
$$

Observe, that $1-2 \gamma^{\prime}=\sqrt{2 \delta-1}$, and that $1-2 \gamma \geq 1-2 \gamma^{\prime}-2 / n>0$ whenever $n$ is sufficiently large. Therefore,

$$
\begin{aligned}
& \frac{2 \gamma \delta-2 \gamma^{\prime} \delta+\gamma^{\prime}-\gamma}{(2 \gamma-1)\left(2 \gamma^{\prime}-1\right)}=\frac{\left(\gamma^{\prime}-\gamma\right) \sqrt{2 \delta-1}}{2 \gamma-1}=\left(\gamma-\gamma^{\prime}\right) \frac{\sqrt{2 \delta-1}}{1-2 \gamma} \leq \\
& \frac{\left(\gamma-\gamma^{\prime}\right) \sqrt{2 \delta-1}}{1-2 \gamma^{\prime}-2 / n} \leq \frac{1}{n}\left(1+\frac{2}{n \sqrt{2 \delta-1}-2}\right)=\frac{1}{n}(1+O(1 / n)) .
\end{aligned}
$$

Above we used the fact that $\gamma \leq \gamma^{\prime}+\frac{1}{n}$. Since $\widetilde{p}(1-\gamma) n$ is not necessarily an integer, we introduce $p_{0}: p_{0}=\lceil\widetilde{p}(1-\gamma) n\rceil /((1-\gamma) n)$. Clearly, the least number of edges one has to remove from the vertices of $A_{l} \cup B_{l}$ in order to find a spanning regular subgraph of $G$ is at least $p_{0}(1-\gamma) n$. With this choice of $p_{0}$ every degree in $A_{l} \cup B_{l}$ will be $\left(1-p_{0}\right)(1-\gamma) n$ after the edge removal process.

Finally, we show that $\left(1-p_{0}\right)(1-\gamma)$ is very close to $\frac{\delta+\sqrt{2 \delta-1}}{2}$ :

$$
\begin{gathered}
\left(1-p_{0}\right)(1-\gamma)-\frac{\delta+\sqrt{2 \delta-1}}{2}=\left(1-p_{0}\right)(1-\gamma)-\left(1-p^{\prime}\right)\left(1-\gamma^{\prime}\right) \leq \\
(1-\widetilde{p})\left(1-\gamma^{\prime}\right)-\left(1-p^{\prime}\right)\left(1-\gamma^{\prime}\right)=\left(1-\gamma^{\prime}\right)\left(1-\widetilde{p}-1+p^{\prime}\right)= \\
\left(1-\gamma^{\prime}\right)\left(p^{\prime}-\widetilde{p}\right)=\left(1-\gamma^{\prime}\right) \frac{1}{n}(1+O(1 / n)) .
\end{gathered}
$$

If $n$ is sufficiently large, then $\left(1-\gamma^{\prime}\right)(1+O(1 / n))<1$, since $0<\gamma^{\prime}<1 / 2$. Hence, if $H \subset G$ is an $r$-regular spanning subgraph, then

$$
\rho_{0} n=\left\lfloor\frac{\delta+\sqrt{2 \delta-1}}{2} n\right\rfloor \leq r<\left(1-p^{\prime}\right)\left(1-\gamma^{\prime}\right) n+1=\frac{\delta+\sqrt{2 \delta-1}}{2} n+1 .
$$

Since $r$ is an integer which is less than $\frac{\delta+\sqrt{2 \delta-1}}{2} n+1$, we get that

$$
\left\lfloor\frac{\delta+\sqrt{2 \delta-1}}{2} n\right\rfloor \leq r \leq\left\lceil\frac{\delta+\sqrt{2 \delta-1}}{2} n\right\rceil \text {, }
$$

and this is what we wanted to prove.

Acknowledgment The author would like to thank Péter Hajnal for the helpful conversations and the anonymous referee for the useful remarks. 


\section{References}

[1] B. Bollobás, A. Saito, N. Wormald, Regular factors of regular graphs, Journal of Graph Theory 9 (1985) 97-103.

[2] B. Csaba, M. Mydlarz, Approximate multipartite version of the Hajnal-Szemerédi theorem, submitted for publication.

[3] B. Csaba, Approximating the Bollobás-Eldridge-Catlin conjecture for bounded degree graphs, manuscript.

[4] M. Krause, A simple proof of the Gale-Ryser theorem, The American Mathematical Monthly 4 (1996) 335-337.

[5] L. Lovász, Combinatorial Problems and Exercises, Akadémiai Kiadó, Budapest, 1993.

[6] H. J. Ryser, Combinatorial mathematics, Wiley and Sons, 1963

[7] W. T. Tutte, The factors of graphs, Canadian J. Math. 4 (1952) 314-328. 